\title{
Chemiluminescence of 4-styrylphthalhydrazides with crown ether as ionophore
}

\author{
Jiro Motoyoshiya,* Msanori Hotta, Yoshinori Nishii, and Hiromu Aoyama
}

Department of Chemistry, Faculty of Textile Science and Technology, Shinshu University, Ueda, Nagano 386-8567, Japan

\begin{abstract}
Two 4-styrylphthalhydrazides bearing crown ether moieties as ionophores were prepared and their chemiluminescence behavior was investigated. Their aerobic oxidation in the presence of excess tert-BuOK in DMSO provided the chemiluminescence with much shorter emission wavelengths than those of the corresponding phthalate dianions, indicating that the emission arose from the excited pthalates incorporated with potassium ions at the ionophore sites. Such incorporation of potassium ions with the crown sites also reduced the fluorescence intensities of the phthalate dianions which caused an energy transfer chemiluminescence, resulting in the additional emissions of the phthalhydrazide monoanions in much longer wavelength regions. On the other hand, only the emission from the excited phthalate dianion was detected under aqueous conditions using aqueous hydrogen peroxide in acetonitrile as the oxidant, but no meaningful difference in the chemiluminescence intensity depending on the kind of metal cations was observed.
\end{abstract}

KEYWORDS: chemiluminescence, phthalhydrazide, crown ether, ionophore

\section{INTRODUCTION}

The chemiluminescence of phthalhydrazides is based on their oxidation reactions that form fluorescent phthalate dianions in the excited states (1). Of a number of various phthalhydrazides, luminol (2-aminophthalhydrazide) is the most popular and frequently used as a chemiluminescent compound, whose noticeable feature is to act as an analytical tools by emitting light as the signal indicating the presence as well as the amount of widespread target substances such as blood for a criminal investigation (2). There have been various extensions of the phthalhydrazide chemiluminescence, many of which are structural modifications not only to enhance the emission efficiency, but also used for determination of certain chemical species $(3,4)$. However, only a few chemiluminophores with ionophores $(5,6)$ have been studied to date, and the potential application as a molecular recognition system is suggested. On the other hand, we have recently reported (7) that 
during the chemiluminescent oxidation of various 4-styrylphthalhydrazides, the emitter and the emission intensity as well as the emission wavelength are highly dependent on the electronic nature of the substituents attached to the styryl groups. These studies prompted us to investigate the chemiluminescence of new 4-styrylphthalhydrazides bearing ionophores such as crown ethers. In this paper, we report their chemiluminescence behavior due to the interaction of the ionophores and metal cations.

\section{MATERIALS AND METHODS}

\section{Preparation of materials}

Two crowned benzaldehydes, 4'-formylbenzo-15-crown-5 and 4'-formylbenzo-18-crown-6, and dimethyl 4-(dimethylphosphono)methylphthalate were prepared according to the established methods (4).

\section{Preparation}

of (E)-4-[2-(6,7,9,10,12,13,15,16-octahydro-5,8,11,14,17-pentaoxabenzocyclo-pentadecen-2-yl)vin yllphthalhydrazide, 1 . To a solution of sodium methoxide $(0.40 \mathrm{~g}, 7.40 \mathrm{mmol})$ and dimethyl 4-(dimethylphosphono)methylphthalate in THF $(20 \mathrm{~mL})$ was added a solution of 4'-formylbenzo-15-crown-5 (0.94 g, $3.16 \mathrm{mmol})$ in THF (10mL). The solution was heated for $4 \mathrm{~h}$ under reflux. After removal of the solvent, the residue was treated with a saturated ammonium chloride solution and ethyl acetate, then the organic layer was washed with brine and dried over magnesium sulfate. After filtration and removal of the solvent, the product was recrystallized from ethyl acetate and hexane to give dimethyl (E)-4-[2-(6,7,9,10,12,13,15,16-octahydro-5,8,11,14,17-pentaoxabenzo-cyclopentadecen-2-yl)vinyl] phthalate (0.79 g, $51 \%) .{ }^{1} \mathrm{H}$ NMR $\left(400 \mathrm{~Hz}, \mathrm{CDCl}_{3}\right): \delta_{\mathrm{H}} 3.73-3.80(\mathrm{~m}, 8 \mathrm{H}), 3.88(\mathrm{~s}, 3 \mathrm{H}), 3.89$ (s, 3H), 3.90-3.93 (m, 4H), 4.17-4.23 (m, 4H), 6.86 (d, 1H), 6.91 (d, 1H, J = $16.2 \mathrm{~Hz}), 7.06$ (d,1H), 7.07 (s, 1H), 7.14 (d, 1H, $J=16.2 \mathrm{~Hz}), 7.58$ (d, 1H), 7.74-7.78 (m, 2H) ppm. To a solution of this phthalate $(0.30 \mathrm{~g}, 0.62 \mathrm{mmol})$ in warm ethylene glycol $(5 \mathrm{~mL})$ was added distilled hydrazine (1.00 $\mathrm{mL}, 3.09 \mathrm{mmol})$. The solution was heated at $120{ }^{\circ} \mathrm{C}$ for $4 \mathrm{~h}$. After cooling at room temperature, hexane and ethanol were added to the reaction mixture and the residue was collected by filtration. The product was washed with petroleum ether and dried under vacuum to give a pale-yellow powder (0.15 g, 49\%). ${ }^{1} \mathrm{H}$ NMR (400 MHz, DMSO-d ${ }_{6}$ ): $\delta_{\mathrm{H}} 3.55-3.65$ (m, 8H), 3.70-3.78 (m, 4H), 3.96-4.06 (m, 4H), 6.77-8.00 (m, 6H), 7.18 (d, 1H, $J=16.4 \mathrm{~Hz}), 7.23$ (d, 1H, $J=16,4 \mathrm{~Hz})$. HRMS 
(EI) calcd for $\mathrm{C}_{24} \mathrm{H}_{26} \mathrm{~N}_{2} \mathrm{O}_{7}\left(\mathrm{M}^{+}\right)$454.1740, found 454.1743 .

Preparation

of

(E)-4-[2-(6,7,9,10,12,13,15,16,18,19-decahydro-5,8,11,14,17,20-hexaoxabenzo-cycloocatadecen-

2-yl)vinyl]phthalhydrazide, 2. The corresponding phthalate and $\mathbf{2}$ were prepared in a manner similar to that described above. Dimethyl (E)-4-[2-(6,7,9,10,12,13,15,16,18,19-decahydro-5,8,11,14,17,20-hexaoxabenzocyclo-ocatadecen-2yl)vinyl]phthalate (0.63 g, $41 \%)$ was obtained from the reaction of 4'-formylbenzo-18-crown-6 (1.00 g, $2.92 \mathrm{mmol})$, dimethyl 4-(dimethylphosphono)methylphthalate (1.11 g, $2.92 \mathrm{mmol})$, and sodium methoxide (0.38 g, $7.02 \mathrm{mmol})$ in THF (30 mL). ${ }^{1} \mathrm{H}$ NMR (400 Hz, $\left.\mathrm{CDCl}_{3}\right): \delta_{\mathrm{H}} 3.59-3.81$ (m, 12H), 3.84-3.97 (m, 4H), 3.90 (s, 3H), 3.93 (s, 3H), 4.14-4.26 (m, 4H), 6.84-7.06 (m, 1H), 6.93 (d, 1H, $J=16.2 \mathrm{~Hz}$ ), 7.13 (d, 1H, $J=16.2 \mathrm{~Hz}), 7.54-7.61$ (m, 1H), 7.70-7.80 (m, 2H) ppm. $2(0.13$ g, $35 \%)$ was obtained from the reaction of this phthalate $(0.35 \mathrm{~g}, 0.65 \mathrm{mmol})$ and hydrazine $(0.21$ $\mathrm{mL}, 6.51 \mathrm{mmol}$ ) in ethylene glycol (5 mL). ${ }^{1} \mathrm{H}$ NMR (400 MHz, DMSO-d $\left.\mathrm{d}_{6}\right): \delta_{\mathrm{H}} 3.42-3.67(\mathrm{~m}, 12 \mathrm{H})$, 3.71-3.86 (m, 4H), 4.07-4.20 (m, 4H), 6.99 (d, 1H),7.18 (d, 1H), 7.36 (s, 1H), 7.39 (d, 1H, $J=16.4$ $\mathrm{Hz}$ ), 7.47 (d, $1 \mathrm{H}, J=16.4 \mathrm{~Hz}$ ), 7.91-8.32 (m, 3H). HRMS (EI) calcd for $\mathrm{C}_{26} \mathrm{H}_{30} \mathrm{~N}_{2} \mathrm{O}_{8}\left(\mathrm{M}^{+}\right)$494.2002, found 498.2002 .

Preparation

of dipotassium (E)-4-[2-(6,7,9,10,12,13,15,16-octahydro-5,8,11,14,17-pentaoxa-benzocyclo-pentadecen-2-yl)vi nyl]phthalate, 3. To a solution of potassium hydroxide $(0.40 \mathrm{~g}, 5.96 \mathrm{mmol})$ in $\mathrm{MeOH}(10 \mathrm{~mL})$ was added the precursory ester $(0.29 \mathrm{~g}, 0.60 \mathrm{mmol})$ in $\mathrm{MeOH}(10 \mathrm{~mL})$ under a nitrogen atmosphere. After the solution was heated under reflux, the precipitates were collected by filtration, washed with $\mathrm{MeOH}$ and petroleum ether, then dried under vacuum. The product was obtained as a pale-yellow powder (0.17 g, 55\%). ${ }^{1} \mathrm{H}$ NMR $\left(400 \mathrm{~Hz}, \mathrm{D}_{2} \mathrm{O}\right): \delta_{\mathrm{H}} 3.40-3.53(\mathrm{~m}, 8 \mathrm{H}), 3.60-3.68(\mathrm{~m}, 4 \mathrm{H})$, 3.85-3.99 (m, 4H), 6.74 (d, 1H), 6.89 (d, 1H, J=16.4 Hz), 6.92-6.98 (m, 2H), 6.98 (d, 1H, J = 16.4 Hz), 7.28 (d, 1H), 7.33 (d, 1H), 7.38 (s, 1H) ppm.

Preparation

of

dipotassium

(E)-4-[2-(6,7,9,10,12,13,15,16,18,19-decahydro-5,8,11,14,17,20-hexa-oxabenzocycloocatadecen-

2-yl)vinyl]phthalhydrazide, 4 . This salt was prepared (0.095 g, $44 \%)$ in a manner similar to that described above using the precursory ester $(0.20 \mathrm{~g}, 0.38 \mathrm{mmol})$ and potassium hydroxide $(0.24 \mathrm{~g}$, $3.58 \mathrm{mmol})$ in $\mathrm{MeOH}(14 \mathrm{~mL}) .{ }^{1} \mathrm{H}$ NMR $\left(400 \mathrm{~Hz}, \mathrm{D}_{2} \mathrm{O}\right): \delta_{\mathrm{H}} 3.35-3.55$ (m, 12H), 3.61-3.75 (m, 4H), 3.78-3.88 (m, 4H), 6.93-7.24 (m, 3H), 7.09 (d, 1H, $J=16.2 \mathrm{~Hz}), 7.18(\mathrm{~d}, 1 \mathrm{H}, J=16.2 \mathrm{~Hz})$, 7.42-7.61 (m, 3H) ppm. 


\section{Measurements of chemiluminescence spectra and quantum yields}

Chemiluminescence spectra. A solution $(1 \mathrm{~mL})$ of the phthalhydrazides in DMSO $\left(1.0 \times 10^{-3} \mathrm{M}\right)$ and a solution $(1 \mathrm{~mL})$ of tert-BuOK $\left(2.0 \times 10^{-2} \mathrm{M}\right)$ were mixed in a quartz cell and the spectra were immediately recorded.

Chemiluminescence quantum yields $\left(\Phi_{\mathrm{CL}}\right)$. The measurements were carried out by a photon-counting method using a Hamamatsu Photonics R464 photomultiplier connected to a photon-counting unit (C3866) and a photon-counting board M8784 according to a previously reported procedure, and the luminal chemiluminescence was used as the standard in DMSO for calibration of the photomultiplier tube (8). For a typical run, a solution of tert-BuOK in DMSO (2.0 $\left.\mathrm{x} 10^{-2} \mathrm{M}\right)$ was added to a solution of 1 in DMSO $\left(1.0 \times 10^{-5} \mathrm{M}\right)$ in a quartz cell placed in front of the photomultiplier and the photons generated over a $2 \mathrm{~h}$ period were counted. The average of the values obtained by a few measurements was used for the calculation of $\Phi_{\mathrm{CL}}$.

\section{RESULTS AND DISCUSSION}

The 4-styrylphthalhydrazides bearing the crown ether moieties were prepared according to a previously reported method as shown in Scheme 1. The Horner-Wittig reaction of the dimethyl (4-dimethylphosphonomethyl)phthalates and two crowned benzaldehydes afforded the dimethyl (E)-4-styrylphthalates, which were heated with hydrazine to give the 4-styrylpthalhydrazides, 1 and 2. The potassium phthalates, $\mathbf{3}$ and $\mathbf{4}$, necessary for identification of the emitters, were obtained by saponification of the corresponding esters.
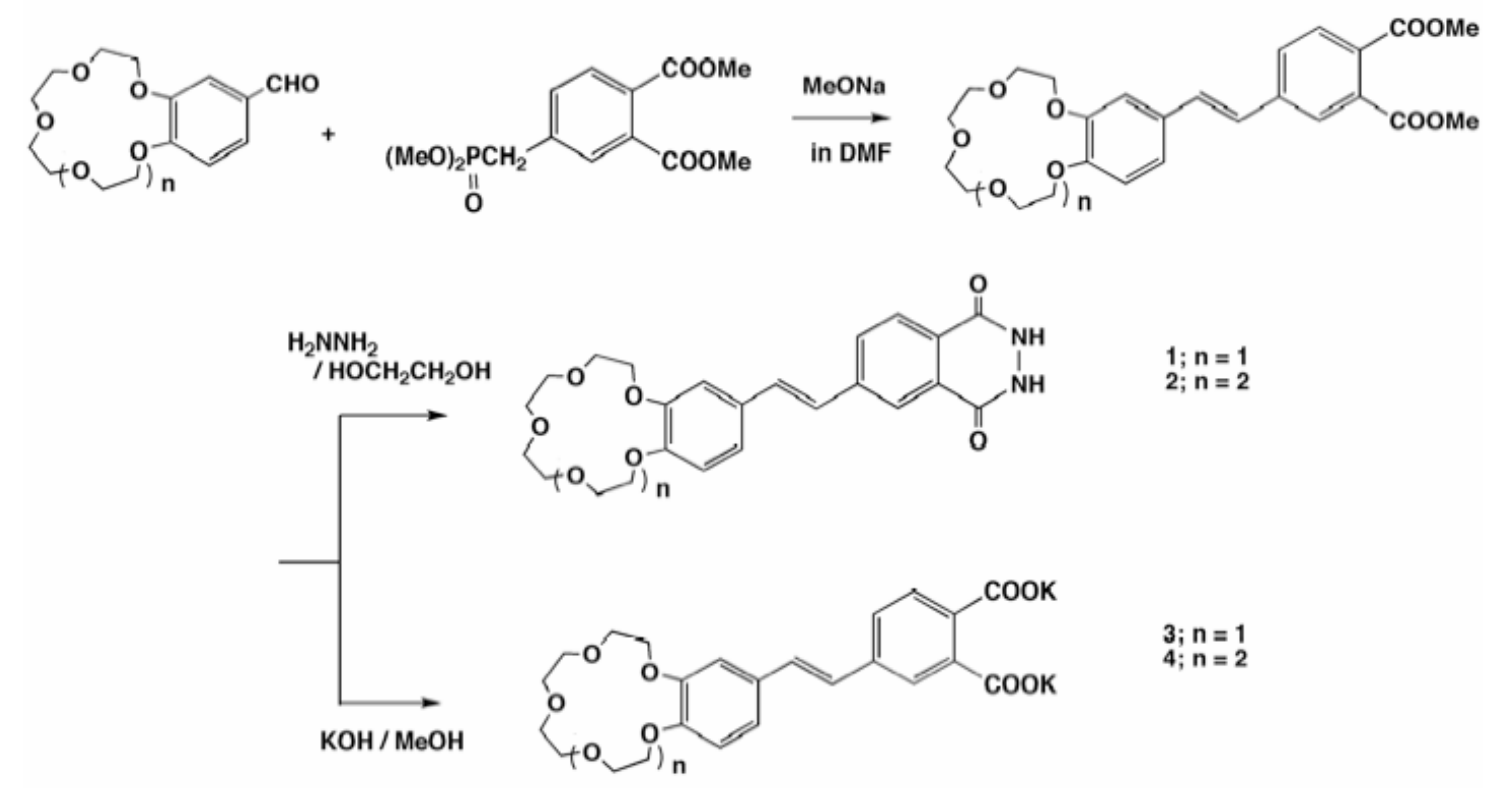
Scheme 1. Preparation of 4-styrylphthalhydrazides and dipotassium 4-styrylphthalates bearing crown ether moieties.

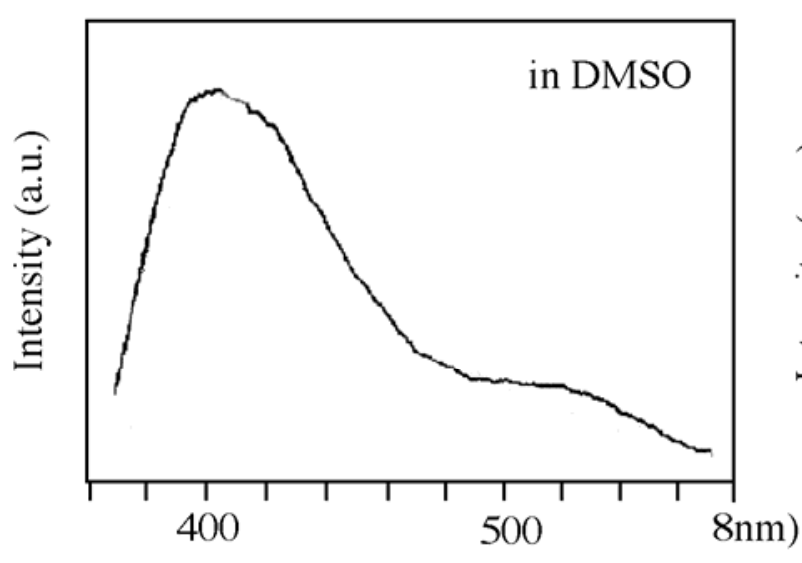

Figure 1A. Chemiluminescence spectrum of the reaction of 1 .

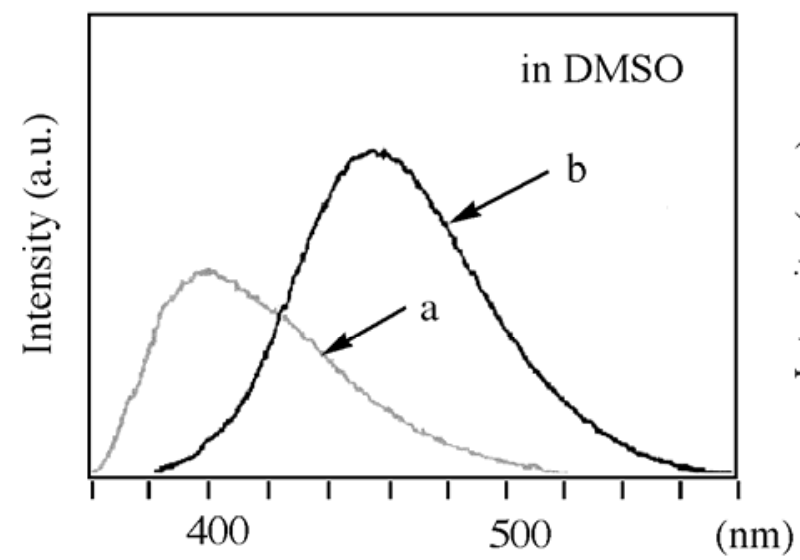

Figure 1C. Fluorescence spectra of $\mathbf{3}$. a: dipotassium phthalate with excess tert-BuOK, b: dipotassium phthalate.

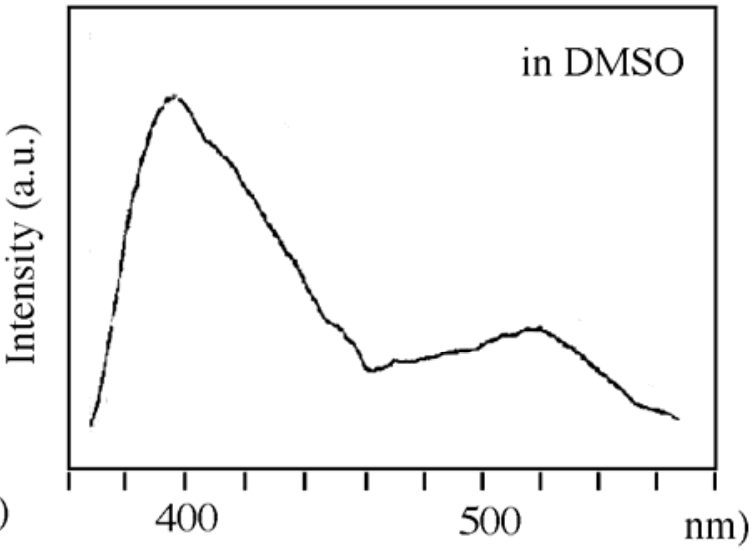

Figure 1B. Chemiluminescence spectrum of the reaction of $\mathbf{2}$.

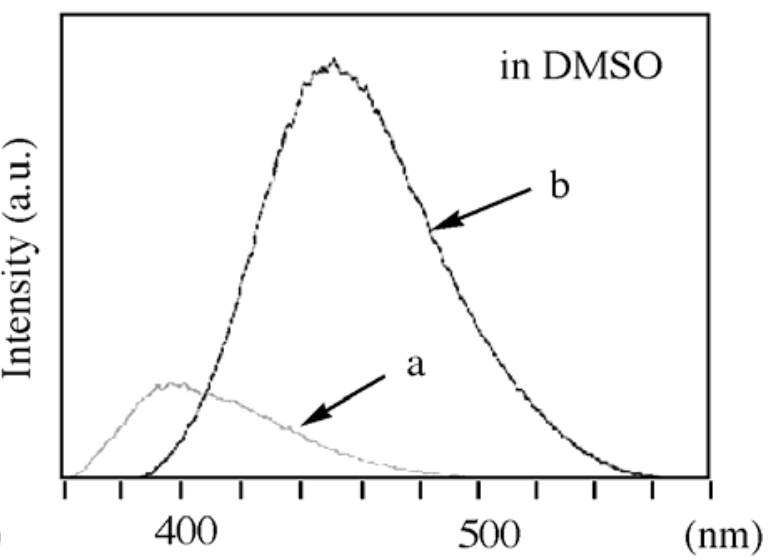

Figure 1D. Fluorescence spectra of 4. a: dipotassium phthalate with excess tert-BuOK, b: dipotassium phthalate.

Treatment of the prepared phthalhydrazides with tert-BuOK in DMSO under aerobic conditions provided the chemiluminescence with the maximum intensities at $400 \mathrm{~nm}$ and $394 \mathrm{~nm}$ for 1 and 2 (Figures 1A and 1B), respectively, which were identical to the fluorescence spectra of the corresponding 4-styrylphthalate dianions, 3 and 4, in the presence of excess tert-BuOK (Figures 1Ca and 1Da). These chemiluminescence spectra around $400 \mathrm{~nm}$ were the emissions from the excited phthalate dianions incorporated with the potassium cations in the crown cavities (Figure 
2A), because both 3 and 4 in DMSO showed their fluorescence spectra at $452 \mathrm{~nm}$ (Figures $1 \mathrm{Cb}$ and $1 \mathrm{Db})$, a much longer wavelength with stronger intensities than that observed in the absence of tert-BuOK. No significant effect of the potassium ion on the fluorescence spectrum of the dipotassium 4-styrylphthalate lacking of an ionophore site supports such an interaction of $\mathbf{1}$ and $\mathbf{2}$ between the crown sites and potassium ions. Interestingly, additional emissions around $520 \mathrm{~nm}$ for both 1 and 2 were detected (Figures 1A and 1B), which were assigned to the emission from their excited monoanions as described below. Previously, we reported (7) that the emission wavelengths for several 4-styrylphthahydrazides were highly dependent on the electronic nature of the substituents attached to the terminal phenyl groups; an electron-donating substituent tends to shift the emission to a longer wavelength region. This is due to the change in the fluorescence character of the emitters, the phthalate dianions. For example, dipotassium 4-styrylpthalate displays its fluorescence spectrum at $363 \mathrm{~nm}$, while 4-(3',4'-dimethoxystyryl)phthalate and 4-(4'-dimethylaminnostyryl)phthalate show the maximum emissions at $444 \mathrm{~nm}$ and $452 \mathrm{~nm}$, respectively. Therefore, the blue-shifts of the chemiluminescence spectra for $\mathbf{1}$ and $\mathbf{2}$ suggest that the electron donating-ability of the crown moieties was controlled by chelation of the potassium ions, namely, such a metal-chelation decreases the electron-donating effect of the crown moiety leading to a decrease in an charge separation in the excited states, resulting in a blue-shift of the emission.

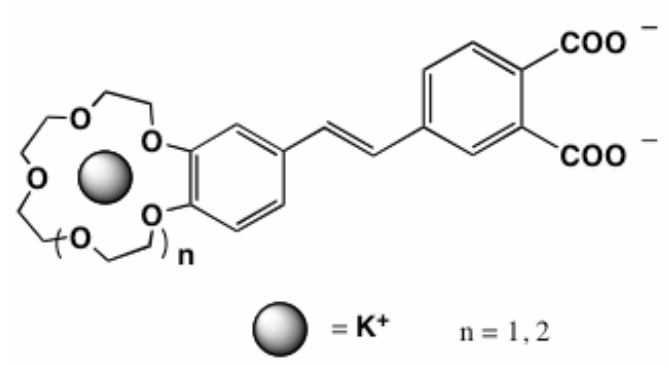

A

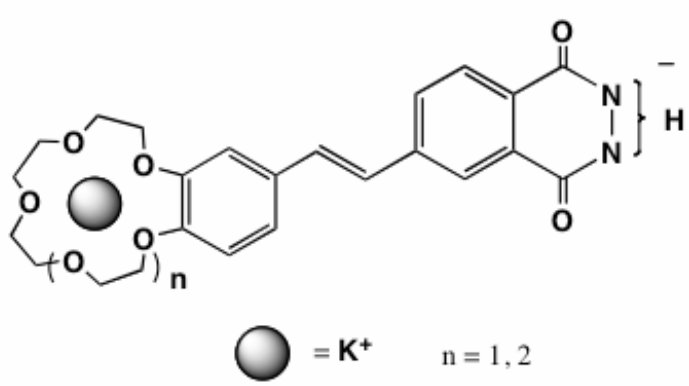

B

Figure 2. The emitters in the chemiluminescence reactions of $\mathbf{1}$ and $\mathbf{2}$.

Although an accurate comparison was difficult because of the complexity of the chemiluminescence of both $\mathbf{1}$ and $\mathbf{2}$ giving two emitters, the relative total chemiluminescence quantum yields of luminol to 1 was almost in the ratio of $1: 0.62$, and 1 exhibited about a 4 times stronger chemiluminescence than $\mathbf{2}$. As mentioned above, the chemiluminescence spectra of $\mathbf{1}$ and $\mathbf{2}$ were accompanied by additional emissions at $520 \mathrm{~nm} 533 \mathrm{~nm}$, respectively, much longer wavelengths than the fluorescence of $\mathbf{3}$ and $\mathbf{4}$. It is known (9) that when a simple phthalhydrazide is subjected to the chemiluminescent reaction, the emitter is not the non-fluorescent phthalte dianion, 
but the excited phthalhydrazide monoanion generated by an energy transfer from the excited phthalate dianion, exhibiting a chemiluminescence at $526 \mathrm{~nm}$. A similar phenomenon has been found in our study using the 4-styrylphthalhydrazides (7); when the phthalate dianion, the final reaction product, was weakly fluorescent, the chemiluminescence reaction of the corresponding phthalhydrazides provided two emitters, one of which was the phthalate dianion and the other phthalhydrazide monoanion. The chemiluminescence accompanied by two emitters in the present study is also the case with emissions around $520 \mathrm{~nm}$ for the reaction of $\mathbf{1}$ and $\mathbf{2}$ that arose from their monoanion as shown in Figure 2B. Thus, binding of the potassium ion into the crown cavity was found to control the chemiluminescence behavior. The emission from the phthalhydrazide monoanion seems to be stronger in the reaction of $\mathbf{2}$ than that of $\mathbf{1}$, which suggests that the crown moiety of $\mathbf{2}$ binds a potassium ion more tightly than that of $\mathbf{1}$. This would be adequate, considering the greater stability constant between the potassium ion and 18-crown-6 than that between potassium ion and 15-crown-5 (10). The possibility of the formation of a 2:1 sandwich complex by two 15-crown-5 moieties and one potassium ion cannot be excluded as previously documented (11).

Next, we explored the chemiluminescence behavior of $\mathbf{2}$ in the presence of various metal cations. To set up the chemiluminescence system without metal cations, the reaction of $\mathbf{2}$ was first carried out using aqueous hydrogen peroxide and tetrabutylammonium hydroxide (TBAH) in acetonitrile. This reaction provided the chemiluminescence at $448 \mathrm{~nm}$, which was in good agreement with the fluorescence spectrum of $\mathbf{4}$ in the same solvents, but showed no other emission peak. The measurements of the chemiluminescence quantum efficiency, $\Phi_{\mathrm{CL}}$, in the presence of LiI, $\mathrm{NaI}, \mathrm{KI}, \mathrm{K}_{2} \mathrm{CO}_{3}$ and $\mathrm{Rb}_{2} \mathrm{CO}_{3}$ were carried out under this oxidation system, and the results are summarized in Table 1. If the crown moiety acts as an ionophore for the metal cations under the above described conditions, the coexisting metal cations would provide the various chemiluminescence intensities. Unfortunately, no significant difference in $\Phi_{\mathrm{CL}}$ depending on the kind of metal ions was detected. The values of $\Phi_{\mathrm{CL}}$ using the metal iodides were ten times smaller than the case in the absence of them, and the decrease in $\Phi_{\mathrm{CL}}$ for the reactions using iodides is not due to the heavy atom effect due to no difference in $\Phi_{\mathrm{CL}}$ between $\mathrm{K}_{2} \mathrm{CO}_{3}$ and $\mathrm{Rb}_{2} \mathrm{CO}_{3}$, but probably due to the decomposition of the peroxide species by the iodide ion with a low redox potential. The use of the carbonates resulted in the almost the same efficiencies as the operation without the additives, but no difference between $\mathrm{K}^{+}$and $\mathrm{Rb}^{+}$was found. The absence of any effect by the ionophore is probably due to the solvation of the metal cations by water that would inhibit the chelation to the crown moiety (12). It is also interesting to note that no effect of the ionophore on the chemiluminescence in the aqueous system is related to the fact that the phthalate dianion was detected as only one emitter, namely, the fluorescence intensity of the phthalate dianion did not decrease due to the absence of the interaction between the potassium ion and the crown site, and 
consequently, strong enough to be an emitter itself. In order to emphasize the difference in the chemiluminescence intensity depending on the kind of metal cations, it will be necessary to use the ionophore that strongly captures metal cations even in water.

\section{CONCLUSION}

The newly prepared 4-styrylphthalhydrazides bearing the crown ether moieties exhibited a chemiluminescence in a much shorter emission wavelength region in the presence of excess amount of potassium ions than was expected for the corresponding phthalate dianions, which is due to the host-guest interaction between the potassium ions and the crown moieties. Binding of the potassium ion in the crown cavity controls the chemiluminescence behavior to provide two emitters, the phthalhydrazide

Table 1. Chemiluminescence of $\mathbf{2}$ in the presence of various metal salts. ${ }^{a}$

\begin{tabular}{ccc}
\hline additive & $\begin{array}{c}\mathrm{CL} \lambda \max \\
(\mathrm{nm})\end{array}$ & $\begin{array}{c}\Phi_{\mathrm{CL}} \times 10^{4} \\
(\text { einstein/mol) }\end{array}$ \\
\hline none & 439 & 4.6 \\
$\mathrm{LiI}$ & 444 & 0.30 \\
$\mathrm{NaI}$ & 438 & 0.32 \\
$\mathrm{KI}$ & 442 & 0.29 \\
$\mathrm{~K}_{2} \mathrm{CO}_{3}$ & 436 & 4.4 \\
$\mathrm{Rb}_{2} \mathrm{CO}_{3}$ & 436 & 4.5 \\
\hline
\end{tabular}

\footnotetext{
${ }^{a}$ Measured in aqeous acetonitrile comtainig $\mathrm{MeOH}$ at $25^{\circ} \mathrm{C}$. [2] $=5.0 \times 10^{-6} \mathrm{M}$, [additive] $=2.5 \times 10^{-2} \mathrm{M}$, $[\mathrm{TBAH}]=\left[\mathrm{H}_{2} \mathrm{O}_{2}\right]=5.0 \times 10^{-2} \mathrm{M}$.
}

monoanions as well as the phthalate dianions. In an aqueous system, the interaction between the metal cation and the crown site is not detectable, the emitter is only the phthalate dianion, and consequently, no significant difference in the chemiluminescence efficiency was detected in the presence of various metal cations.

\section{Acknowledgements}


One of the authors (JM) is thankful for the financial support by a Grant-in-Aid, from the Ministry of Education, Culture, Sports, Science and Technology of Japan.

\section{REFERENCES}

1. White EH, Roswell DF. The chemiluminescence of organic hydrazides. Acc. Chem. Res. 1970; 3: 54-62.

2. Zhang Z, Zhang S, Zhang X. Recent development and application of chemiluminescence sensors. Anal. Chim. Acta, 2005; 541: 37-47.

3. Tominaga Y, Yoshioka N, Kataoka S, Shigemitsu Y, Hirota T, Sasaki K. Synthesis and chemiluminescence of 1-hydroxy- and 10-aminopyridazino[4,5-b]quinoline-1,4(2H, 3H)-diones. Heterocycles, 1999; 50: 43-46.

4. Creramer JI, Quickenden TI, Apanah MV, Kerr KA, Robertson P. Luminescence, 2003; 18: 193-

5. Okamoto H, Owari M, Kimura M, Satake K. Preparation of a crown-ether-modified lophine peroxide as a guest-sensitive novel chemiluminophore and modulation of its chemiluminescence by metal cations. Tetrahedron Lett. 2001: 42: 7453-7455.

6. . Okamoto H, Kimura M. Preparation of an azacrowned isoluminol and its chemiluminescence initiated by alkali-metal iodides in acetonitrile. Chem. Lett. 2005; 34: 1452-1453.

7. Motoyoshiya J, Yokota K, Fukami S, Konno S, Yamamoto A, Hotta M, Koike R, Yoshioka S, Nishii Y, Aoyama H. Synthesis of 4-styrylphthalhydrazides and their chemiluminescence reaction. Emitters and chemiluminescence efficiency highly dependent on electronic nature of styryl groups. J. Heterocyclic Chem. 2005; 42: 1063-1068.

8. Motoyoshiya J, Sakai N, Imai M, Yamaguchi Y, Koike R, Takaguchi Y, Aoyama H. Peroxyoxalate chemiluminescence of $\mathrm{N}, \mathrm{N}$-bistosyl-1H,4H-quinoxaline-2,3-dione and related compounds. Dependence on electronic nature of fluorophores. J Org, Chem. 2002; 67: 7314-7318.

9. White EH, Roswell DF, Zafiriou OC. The anomalous chemiluminescence of phthalic hydrazide. $J$. Org. Chem. 1969; 34: 2462-2468.

10. Gokel GW, Leevy WM, Weber ME. Crown ethers: Sensors for ions and molecular scaffolds for materials and biological models. Chem. Rev. 2004; 104: 2723-2750.

11. Mallinson PR, Truter MR. Crystal structures of complexes between alkali metal salts and cyclic polyethers. V. 1:2 Complex formed between potassium iodide and 2,3,5,6,8,9,11,12-octa-hydro-1,4,7,10,13-benzopentaoxacyclopentadecin (benzo-1k-crown-5). J. Chem. Soc. Perkin Trans. 2. 1972; 1818-1823. 
12. Piekarski H, Jozwiak M. Thermochemistry of interactions of $\mathrm{Na}^{+}$with benzo-15-crown-5 ether in acetonitrile-water mixtures at 298.5 K. J. Thermal Anal. 1997; 48: 1283-1291. 